\title{
Upregulated SOX9 expression indicates worse prognosis in solid tumors: a systematic review and meta-analysis
}

\author{
Haihua Ruan ${ }^{1}$, Shuangyan Hu${ }^{1}$, Hongyu Zhang ${ }^{1}$, Gang $\mathrm{Du}^{1}$, Xiaoting $\mathrm{Li}^{2}$, Xiaobo $\mathrm{Li}^{2}$ \\ and Xichuan Li ${ }^{1,2}$ \\ ${ }^{1}$ Tianjin Key Laboratory of Food Science and Biotechnology, College of Biotechnology and Food Science, Tianjin University \\ of Commerce, Tianjin, China \\ ${ }^{2}$ Department of Immunology, Tianjin Medical University, Tianjin, China
}

Correspondence to: Xichuan Li, email: xichuanli@tmu.edu.cn Hongyu Zhang, email: zhanghongyu@tjcu.edu.cn

Keywords: solid tumors, SOX9, prognosis, meta-analysis

Received: August 23, $2017 \quad$ Accepted: October 05, $2017 \quad$ Published: November 06, 2017

Copyright: Ruan et al. This is an open-access article distributed under the terms of the Creative Commons Attribution License 3.0 (CC BY 3.0), which permits unrestricted use, distribution, and reproduction in any medium, provided the original author and source are credited.

\section{ABSTRACT}

It was recently reported that increased SOX9 expression drives tumor growth and promotes cancer invasion during human tumorigenicity and metastasis. However, the prognostic value of SOX9 for the survival of patients with solid tumors remains controversial. The present meta-analysis was thus performed to highlight the link between dysregulated SOX9 expression and prognosis in cancer patients. A systematic literature search was conducted using the electronic databases PubMed, Web of Science and Embase to identify eligible studies. A random-effects meta-analytical model was employed to correlate SOX9 expression with overall survival (OS), diseasefree survival (DFS) and clinicopathological features. In total, 17 studies with 3307 patients were eligible for the final analysis. Combined hazard ratios (HRs) and $95 \%$ confidence intervals (CIs) suggested that high SOX9 expression has an unfavourable impact on OS (HR $=1.66,95 \%$ CI 1.36-2.02, $P<0.001)$ and DFS (HR $=3.54,95 \%$ CI 2.29-5.47, $P=0.008$ ) in multivariate analysis. Additionally, the pooled odds ratios (ORs) indicated that SOX9 over-expression is associated with large tumor size, lymph node metastasis, distant metastasis and a higher clinical stage. Overall, these results indicated that SOX9 over-expression in patients with solid tumors might be related to poor prognosis and could serve as a potential predictive marker of poor clinicopathological prognosis factor.

\section{INTRODUCTION}

SOX9 is a member of SOX [SRY (sex determining region Y)-related high mobility group (HMG) box] family and serves as a transcription factor that plays a central role in the development and differentiation of multiple cell lineages [1]. Discovery of SOX9 began with its function underlying campomelic dysplasia (CD), a rare genetic disorder characterized by bowing of the long bones [1]. In the past decade, the knowledge of SOX9 has developed rapidly. SOX9 plays a versatile role in chondrogenesis and skeletal development, in male gonad genesis, in differentiation of multiple organs, in ectoderm development, and in various solid tumors [2-7].
Increased SOX9 expression drives prostate cancer (PCa) tumor growth and angiogenesis and promotes prostate cancer invasion by reactivating the $\mathrm{WNT} / \beta$ catenin signaling that mediates ductal morphogenesis in fetal prostate [8]. SOX9 overexpression significantly induces the proliferation and tumorigenicity of human esophageal squamous cell cancer (ESCC) cells by increasing the expression of phosphorylated Akt and its downstream targets such as phosphorylated forkhead box $\mathrm{O}$ (FOXO) 1 and phosphorylated FOXO3, two members of FOXO family of transcription factors [9]. Aberrant SOX9 expression contributes to the development of gastric cancer by inactivation of GKN1 as an early event [10]. Conversely, knockdown of SOX9 suppresses chondrosarcoma growth 
and migration [11], and induces apoptosis, cell cycle arrest as well as decreased expression of cancer stem cell markers [12-14]. Therefore, inhibited tumor growth and invasion by SOX9 knockdown shed light on regarding SOX9 as a therapeutic target for cancer. A plenty of studies investigated the correlation between SOX9 expression and prognosis in cancer patients, and demonstrated that upregulated expression of SOX9 in malignant tumors was correlated with poor prognosis in patients with different types of solid tumors such as chordoma [13], osteosarcoma [14-16], colorectal carcinoma [17, 18], esophageal squamous cell carcinoma [10, 19], breast cancer [20-23], hepatocellular carcinoma (HCC) [24, 25], glioma [26], chondrosarcoma [27], gastric cancer [28-30], melanoma [31], pancreatic ductal adenocarcinoma (PDAC) [32], ovarian cancer (OC) [33], prostate cancer [34, 35] and non-small cell lung cancer (NSCLC) [36]. However, some other studies revealed that overexpression of SOX9 was not significantly associated with prognosis of some patients with gastric cancer [9] and with breast cancer when looking at overall or 5-year survival [37]. Taken together, the exact clinical and prognostic merit of SOX9 overexpression in various solid tumors remains unclear. Moreover, most of these studies included only a limited number of patients, and the results of each individual study were not conclusive.

In this study, we herein issued a comprehensive meta-analysis to appraise the prognostic significance of SOX9 overexpression in solid human tumors, and illustrate the clinical value of SOX9 as a prognostic indicator and potential therapeutic target for malignant tumor patients.

\section{RESULTS}

\section{Study search information}

The initial search identified 721 publications, of which, 30 studies were of acceptable relevance. However, eight of these studies were excluded because the absence of survival data, and five were excluded because of the absence of information about distinct data. Ultimately, 17 studies met the eligibility criteria and were included in the current meta-analysis (Figure 1).

\section{Description of the studies}

The main characteristics of the 17 identified studies were presented in Table 1. In total, 3307 patients from five regions (China, Korea, United States of America, Australia and Japan) with 11 distinct cancers, chordoma [13], osteosarcoma [14, 16], esophageal cancer [9, 19], hepatocellular carcinoma [24, 38], intrahepatic cholangiocarcinoma [39], pancreatic ductal adenocarcinoma [32], prostate cancer [34, 35], thyroid carcinoma [40], colorectal cancer $[18,41]$, gastric cancers
$[10,42]$, non-small cell lung cancer [36] were included in these studies.

\section{Correlations between SOX9 expression and OS}

The pooled hazard ratio (HR) revealed that overexpressed SOX9 was significantly associated with poor overall survival (OS) for cancer victims in multivariate analysis (HR: 1.66, 95\% CI: 1.36-2.02; Figure 2). However, a significant heterogeneity $\left(\mathrm{I}^{2}=62.5 \%, P=\right.$ 0.001 ) was observed when using a random-effects model to analyze the pooled HR of the OSs.

To minimize heterogeneity, the subgroup analyses were performed according to the ethnics (Asian or not), case number ( $\geq 100$ or not), NOS score $(\geq 7$ or not), follow-up time ( $\geq 120 \mathrm{~m}$ or not), antibody (various company), cut-off value (various scoring criteria). The pooled HRs and heterogeneities according to all these factors were presented in Table 2. Unfortunately, all these subgroup analyses demonstrated that there were no significant lower $\mathrm{I}^{2}$ value when the $P<0.05$. Therefore, subgroup analysis were failed to find the origin of high heterogeneity.

\section{Correlations between SOX9 expression and DFS}

A significant correlation between over-expressed SOX9 and disease-free survival (DFS) was also observed in the patients with solid tumors in multivariate analysis (HR: 3.54, 95\% CI: 2.29-5.47; Figure 3) in the randomeffects model with a significant heterogeneity $\left(\mathrm{I}^{2}=68.1 \%\right.$, $P=0.008)$.

\section{Correlations between SOX9 expression and clinicopathological parameters}

The clinical and pathological parameters collected from the eligible studies were presented in Supplementary Table 1. Meanwhile, pooled results of the correlations were identified between over-expressed SOX9 and clinicopathological features of patients with solid tumors. No significant correlations between over-expressed SOX9 with gender and tumor differentiation were observed. However, the expression of SOX9 was positively associated with tumor size (OR: 1.58, 95\% CI: 1.31-1.91), lymph node metastasis (OR: 1.61, 95\% CI: 1.30-1.99), distant metastasis (OR: $1.53,95 \%$ CI: $1.25-1.87$ ) and a higher clinical stage (OR: $1.68,95 \%$ CI: $1.33-2.12$ ) in the random-effects model with significant heterogeneities (see Table 3 and Supplementary Figure 1).

\section{Assessment of heterogeneity and sensitivity}

There was significant heterogeneities $\left(\mathrm{I}^{2}>50 \%\right)$ between studies in OS and DFS analyses. So the randomeffect model was therefore adopted in these studies. A meta- 
Table 1: Main characteristics of studies exploring the relationship between SOX9 expression and tumor prognosis

\begin{tabular}{|c|c|c|c|c|c|c|c|c|c|c|}
\hline Author & Year & Region & Cancer Type & $\begin{array}{l}\text { Stage / } \\
\text { Grade }\end{array}$ & $\begin{array}{c}\text { No. of } \\
\text { Patients }\end{array}$ & $\begin{array}{l}\text { Follow-up Time } \\
\text { Median (range) }\end{array}$ & Detection Method & Cut-off & $\begin{array}{l}\text { NOS } \\
\text { Score }\end{array}$ & Outcomes \\
\hline Chen H [13] & 2017 & USA & Chordoma & I-III & 50 & $4-250 \mathrm{~m}$ & IHC(Santa Cruz) & $\mathrm{PS}>2$ & 5 & OS, DFS \\
\hline Qi J [14] & 2017 & China & Osteosarcoma & I-III & 97 & $10-72 \mathrm{~m}$ & IHC(Santa Cruz) & IRS $>5$ & 6 & OS \\
\hline Yang Z [19] & 2016 & Korea & Esophageal cancer & $\mathrm{I}-\mathrm{V}$ & 127 & $1-120 \mathrm{~m}$ & IHC(Abnova) & NR & 6 & OS, DFS \\
\hline Liu C [24] & 2016 & China & Hepatocellular Carcinoma & I-III & 148 & $1-80 \mathrm{~m}$ & IHC(Millipore) & $\mathrm{PS}>2$ & 6 & OS \\
\hline Hong Y [9] & 2015 & China & Esophageal cancer & $\mathrm{I}-\mathrm{V}$ & 155 & $1-100 \mathrm{~m}$ & IHC(Abcam) & IRS $>6$ & 7 & OS \\
\hline Matsushima H [39] & 2015 & Japan & $\begin{array}{c}\text { Intrahepatic } \\
\text { cholangiocarcinoma }\end{array}$ & $\mathrm{I}-\mathrm{V}$ & 43 & $1-150 \mathrm{~m}$ & IHC(Abcam) & NR & 5 & OS \\
\hline Xia S [32] & 2015 & China & $\begin{array}{l}\text { Pancreatic ductal } \\
\text { adenocarcinoma }\end{array}$ & $\mathrm{I}-\mathrm{V}$ & 88 & $1-60 \mathrm{~m}$ & IHC(Millipore) & IRS $>6$ & 6 & OS \\
\hline Qin GQ [34] & 2014 & China & Prostate cancer & $\mathrm{T} 2 \mathrm{~A}$ & 98 & $1-140 \mathrm{~m}$ & IHC(Santa Cruz) & $\mathrm{PS}>1$ & 7 & OS, DFS \\
\hline Zhu H [16] & 2013 & China & Osteosarcoma & II-III & 166 & $10-152 \mathrm{~m}$ & IHC(Santa Cruz) & IRS $>5$ & 6 & OS, DFS \\
\hline Yun JY [40] & 2013 & Korea & Thyroid carcinoma & $\mathrm{I}-\mathrm{V}$ & 158 & $47.5 \mathrm{~m}$ for median & IHC(Abnova) & PS $>1$ & 7 & OS \\
\hline Candy P [41] & 2013 & Australia & Colorectal cancer & I-III & 1056 & $69.7 \mathrm{~m}$ for median & IHC(Santa Cruz) & $>50 \%$ & 8 & OS \\
\hline Choi YJ [10] & 2013 & Korea & Gastric cancers & NR & 185 & $1-60 \mathrm{~m}$ & IHC(Millipore) & $>30 \%$ & 7 & OS \\
\hline Zhong WD [35] & 2012 & China & Prostate cancer & $\mathrm{T} 2 \mathrm{~A}$ & 147 & $3-12 y$ & IHC(Santa Cruz) & IRS > 4 & 6 & DFS \\
\hline Guo X [38] & 2012 & China & Hepatocellular Carcinoma & $\mathrm{I}-\mathrm{V}$ & 130 & $\begin{array}{l}8.6 \text { year for } \\
\text { median }\end{array}$ & IHC(Santa Cruz) & IRS $>5$ & 7 & OS, DFS \\
\hline Zhou CH [36] & 2012 & China & Non-small cell lung cancer & $\mathrm{I}-\mathrm{V}$ & 89 & $1-60 \mathrm{~m}$ & IHC(Millipore) & IRS $>6$ & 6 & OS \\
\hline Sun M [42] & 2012 & China & Gastric cancer & NR & 382 & $1-3000 \mathrm{~d}$ & IHC(Millipore) & IRS $>5$ & 8 & OS \\
\hline Lü B [18] & 2008 & China & Colorectal Cancer & $\mathrm{I}-\mathrm{V}$ & 188 & $1-12.5 \mathrm{y}$ & IHC(Santa Cruz) & $\mathrm{PS}>2$ & 7 & OS \\
\hline
\end{tabular}

NR: Not Reported; y: year; m: month; d: day; OS: Overall Survival; DFS: Disease-Free Survival. PS: Percentage Score; IRS: Immunoreactive Score.

regression analysis with published country, case number $(\geq$ 100 or not), antibody (used for different companies) and cut-off value (scores or not) as covariates was conducted. All covariates were fit into the meta-regression model one at a time to identify potential sources of heterogeneity. In terms of OS and DFS, none of these covariates were verified as a significant source of heterogeneity (Table 4). Also, by successively omitting each study from the aggregated survival meta-analyses, sensitivity analysis was performed to evaluate the influence of each individual study on the pooled HR of OS and DFS (Figure 4). The results revealed that the pooled estimates of the effect of over-expressed SOX9 on the OS and DFS of patients with solid tumors did not vary substantially with the exclusion of any individual study, which implies that the results of this meta-analysis are stable.

\section{Publication bias}

We constructed funnel plots and performed Begg's test to assess publication bias. As a result, the shape of the funnel plot for the OS, DFS and clinicopathological parameters seemed symmetrical in the multivariate



Figure 1: Flow diagram of the selection of eligible studies. 
Table 2: Associations between SOX9 expression and OS stratified according to the ethnics, case number, NOS score, follow-up time, antibody and cut-off value

\begin{tabular}{|c|c|c|c|c|}
\hline Categories & Subgroups & Ref & HR (95\% CI) & $\begin{array}{l}\text { Heterogeneity test }\left(\mathrm{I}^{2},\right. \\
P \text {-value) }\end{array}$ \\
\hline \multirow[t]{2}{*}{ Ethnics } & Asian & {$[9,10,14,16,18,19,24,32,34-36,38-$} & $1.98(1.50-2.62)$ & $53.8 \%, 0.009$ \\
\hline & Not Asian & $40,42]$ & $1.19(0.96-1.48)$ & $60.7 \%, 0.111$ \\
\hline \multirow[t]{3}{*}{ Case Number } & $\geq 100$ & {$[13,41]$} & $1.60(1.29-1.99)$ & $71.8 \%, 0.000$ \\
\hline & & {$[9,10,18,19,24,32,34,35,38,39,42]$} & & \\
\hline & $<100$ & {$[13,14,16,36,40,41]$} & $2.05(1.30-3.23)$ & $0.0 \%, 0.770$ \\
\hline \multirow[t]{2}{*}{ NOS Score } & $\geq 7$ & {$[9,10,18,34,38,40-42]$} & $1.41(1.10-1.79)$ & $67.5 \%, 0.003$ \\
\hline & $<7$ & {$[13,14,16,19,24,32,35,36,39]$} & $2.69(1.99-3.62)$ & $46.4 \%, 0.071$ \\
\hline \multirow[t]{2}{*}{ Follow-up Time } & $\geq 120 \mathrm{~m}$ & {$[13,16,18,19,34,35,39]$} & $2.26(1.46-3.50)$ & $78.0 \%, 0.001$ \\
\hline & $<120 \mathrm{~m}$ & {$[9,10,14,24,32,36,38,40-42]$} & $1.53(1.23-1.91)$ & $67.1 \%, 0.001$ \\
\hline \multirow[t]{4}{*}{ Antibody } & Santa Cruz & {$[13,14,16,18,34,35,38,41]$} & $1.58(1.28-1.95)$ & $74.0 \%, 0.001$ \\
\hline & Millipore & {$[10,24,32,36,42]$} & $1.54(0.92-2.59)$ & $41.1 \%, 0.147$ \\
\hline & Abcam & {$[9,39]$} & $3.54(2.11-5.94)$ & $0.0 \%, 0.749$ \\
\hline & Abnova & {$[19,40]$} & $1.63(0.94-2.83)$ & $0.0 \%, 0.573$ \\
\hline \multirow[t]{4}{*}{ Cut-off Value } & IRS & {$[9,14,16,32,35,36,38,42]$} & $2.64(1.67-4.17)$ & $30.3 \%, 0.197$ \\
\hline & PS & {$[13,18,24,34,40]$} & $1.47(0.99-2.18)$ & $0.0 \%, 0.760$ \\
\hline & Percentage & {$[10,41]$} & $1.13(0.92-1.38)$ & $0.0 \%, 0.844$ \\
\hline & NR & {$[19,39]$} & $2.08(1.37-3.16)$ & $0.0 \%, 0.366$ \\
\hline
\end{tabular}

m: month; PS: Percentage Score; IRS: Immunoreactive Score; NR: Not Reported.

analysis method (Figure 5 and Supplementary Figure 2). The Begg's and Egger's tests revealed non-significant values $(P=0.322$ and 0.08 , respectively).

\section{DISCUSSION}

The transcription factor SOX9 is a member of SOX family proteins which contain a highly conserved HMG domain that was first identified in Sry, an essential factor involved in mammalian male sex determination [43]. In general, proteins containing a domain with $50 \%$ or higher amino acid similarity to the HMG are referred to as SOX proteins. Around 20 SOX proteins have been confirmed in mice and humans, and are grouped A through $\mathrm{H}$ based on the structural homology outside of their HMG boxes. SOX9 belongs to SoxE proteins [44] and exerts its function in sex determination, cell differentiation during embryonic development, and cell maintenance and specification during adult life of mice and human [2].

Since the first record on the analysis of SOX9 expression in human cancer published in 1997 [45], more than hundreds studies have explored the role of SOX9 expression in tumors in larger patient groups. SOX9 is over-expressed in various human malignancies and growing evidence demonstrates its association with human solid tumor growth $[9,10]$. Conversely, knockdown of SOX9 provides inhibition of chondrosarcoma growth and migration, and induces apoptosis and cell cycle arrest [26]. The meta-analysis presented herein is the first comprehensive description of all reported survival data from 3307 solid tumor patients from 17 eligible studies, which met the inclusion criteria, investigating the impact of SOX9 expression in human tumors on prognosis. For all studies, SOX9 expression was detected by IHC. By meta-analysis of the 17 studies, we identified the pool HRs which indicated that SOX9 was a factor in poor prognosis in various cancers. Because there is no significant heterogeneity among our included studies, so we did not perform further subgroup analyses.

For the reasons of SOX9 overexpression correlated with poor prognosis in various solid tumors, we summarized as follows: i) Downregulated expression of E-cadherin and increased expression of $\beta$ catenin, which are key factors for epithelial-mesenchymal transition (EMT) in gastric cancers, by SOX9 overexpression. Aberrant SOX9 expression inactivates the activity of gastrokine 1 (GKN1) [46]. Inactivation of GKN1 downregulates expression of E-cadherin and increases expression of $\beta$ catenin in gastric cancers [46]. Besides that, SOX9 activates TGF $\beta /$ Smad signaling, activation of this signaling pathway upregulates snail expression, which in turn triggers EMT, resulting in down-regulation of E-cadherin and increased expression of $\beta$ catenin [47]. Overexpression of $\beta$ catenin leads to the induction of EMT in gastric cancers and partially restores the colonyforming potential in squamous cell cancer (SCC) development [48]. ii) SOX9 is important in maintaining the properties of cancer stem cell (CSC) in various tumors. The hedgehog $(\mathrm{Hh})$ pathway is involved in CSC maintenance in various tumors [49]. Glioma-associated oncogene homolog 1 (Gli1) is a key mediator of the Hh pathway; involved in CSC maintenance [49]. Gli1 expression is correlated with 
Table 3: Meta-analysis results of the associations of increased SOX9 expression with clinicopathological parameters

\begin{tabular}{llll}
\hline Clinicopathological parameter & Ref & Overall OR (95\% CI) & Heterogeneity test $\left(\mathbf{I}^{2}, \boldsymbol{P}\right.$-value) \\
\hline Gender (male vs female) & {$[9,14,16,18,19,32,38,39]$} & $0.99(0.85-1.15)$ & $0.0 \%, 0.439$ \\
Tumor Differentiation (poor VS well) & {$[9,18,19,32,38,39]$} & $1.13(0.93-1.39)$ & $59.2 \%, 0.031$ \\
Tumor Size (T3-4 vs T1-2) & {$[9,14,16,18,19,32,38,39]$} & $1.58(1.31-1.91)$ & $81.3 \%, 0.000$ \\
Lymph Node Metastasis (yes vs no) & {$[9,18,19,32,39]$} & $1.61(1.30-1.99)$ & $84.9 \%, 0.000$ \\
Distant Metastasis (yes vs no) & {$[9,14,16,19,32,39]$} & $1.53(1.25-1.87)$ & $27.3 \%, 0.230$ \\
Clinical Stage (III-IV vs I-II) & {$[9,14,16,18,19,32,39]$} & $1.68(1.33-2.12)$ & $90.4 \%, 0.000$ \\
\hline
\end{tabular}

Table 4: Results of meta-regression analysis exploring source of heterogeneity with OS and DFS

\begin{tabular}{lcccccc}
\hline \multirow{2}{*}{ Covariates } & \multicolumn{3}{c}{ OS } & \multicolumn{3}{c}{ DFS } \\
\cline { 2 - 7 } & Coef. & S.E. & P value & Coef. & S.E. & $\boldsymbol{P}$ value \\
\hline Country & -0.129 & .083 & 0.144 & -0.310 & 0.328 & 0.399 \\
Case Number & 0.263 & 0.261 & 0.331 & 0.227 & 0.576 & 0.713 \\
Antibody & 0.033 & 0.122 & 0.792 & -0.250 & 0.103 & 0.071 \\
Cut-off value & -0.065 & 0.121 & 0.599 & 0.310 & 0.123 & 0.065 \\
\hline
\end{tabular}

Coef.: Coefficient; S.E.: Standard Error;

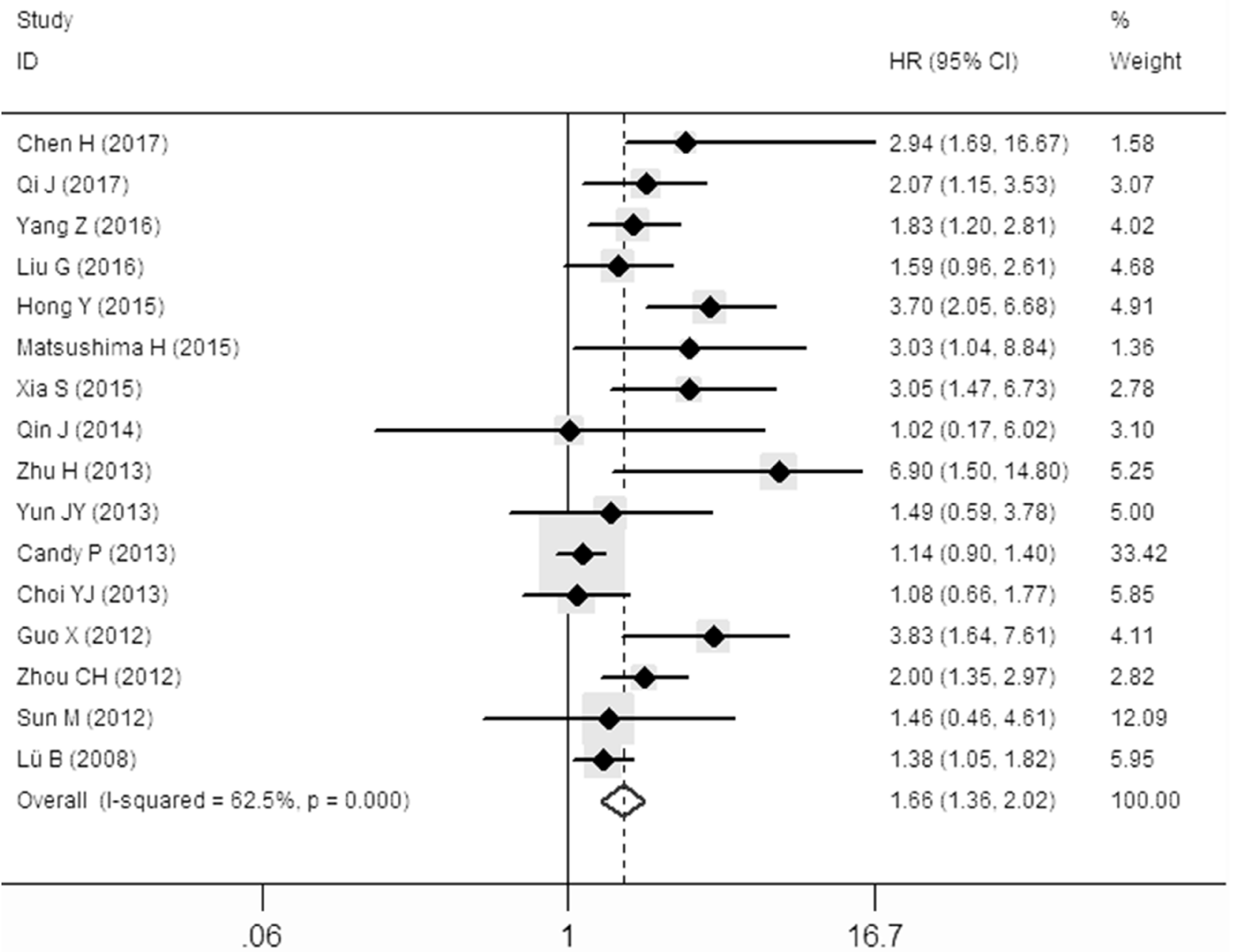

Figure 2: Forest plot describing the association between over-expressed SOX9 and OS. 
the expression of stemness genes, SOX9, and cell cycle regulators such as p21, cyclin D1, cyclin E1, and NF$\kappa \mathrm{B}$, which are strongly linked to worse clinical outcome and independent poor prognostic factors in overall survival and disease-free survival in ESCC [19]. iii) Enhanced transcription of SOX9 responsive genes during tumorgenecity. SOX9 is showed to bind to 4293 genes in common between the mouse and bovine genomes [50]. Most of these genes are already known to be involved in sex determination. Moreover, transcriptomic (RNA-seq) analysis of foetal testes from SOX9 knockout mice showed that SOX9 not only regulates transcription of its target genes directly, but also influences their RNA splicing [50]. Thus, in great possibility, the overexpressed SOX9 might results in disordered gene expression in tumorgenecity. For example, SOX9 transcriptionally activated FOXK2, which belongs to the fork head DNA binding protein family, has been shown to play a critical role in tumorigenesis, high expression of FOXK2 is significantly correlated with poor survival of colorectal cancer [51]. iv) SOX9 promotes osteosarcoma (OS) cell growth by inhibiting the promoter activity of the CLDN8 gene and down-regulating CLDN8 expression, which functions as an oncogenic factor and was up-regulated in OS cells [14]; Overexpression of SOX9 in adult mouse prostate epithelia induces an early high-grade prostate intraepithelial neoplasia (PIN) lesion, indicating that SOX9 augments the loss of PTEN, which is a factor vital for tumor formation [52].
Additionally, no publication bias was observed. Our meta-analysis results involve several important implications. First, it shows that over-expressed SOX9 was positively related to poor OS and DFS in solid tumor patients. Second, pooled results of the correlations were identified between over-expressed SOX9 and clinicopathological features of patients with solid tumors, indicating that SOX9 may serve as a promising therapeutic target. Third, our results showed the expression of SOX9 was positively associated with lymph node metastasis, large tumor size, distant metastasis and a higher clinical stage. We can explain this result by SOX9's ability to enhance prostate cancer $(\mathrm{PCa})$ tumor growth, promote tumor cell proliferation, invasion and metastasis [31]. Because of its involvement in these processes, SOX9 is likely to be causally involved in tumor progression and, consequently, increased levels of SOX9 would be expected to indicate a poor prognosis. Finally, it highlights the potential clinical application of SOX9 as a valuable prognostic biomarker.

This meta-analysis was properly performed, however, further analysis with several limitations would be considered in the future. Firstly, need more trials to analysis; second, some of the survival data were extracted from Kaplan-Meier curves and might be less reliable than a direct analysis of variance; third, we need to search more non-English publications. In addition, the possible existence of unpublished studies could also result in
Study

ID
$\%$

$\operatorname{HR}(95 \% \mathrm{Cl}) \quad$ Weight

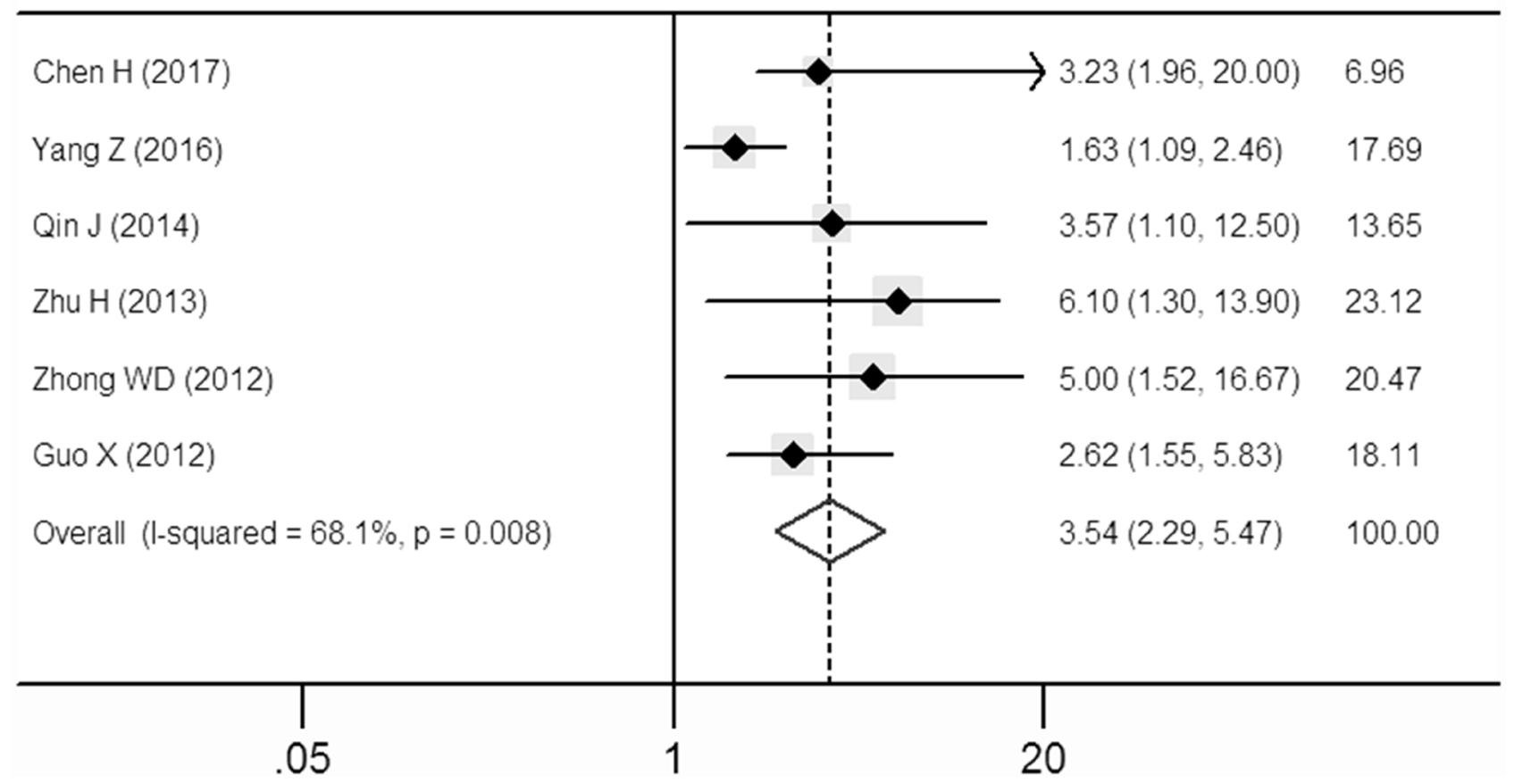

Figure 3: Forest plot describing the association between over-expressed SOX9 and DFS. 
Sensitivity analysis of OS



B

Sensitivity analysis of DFS

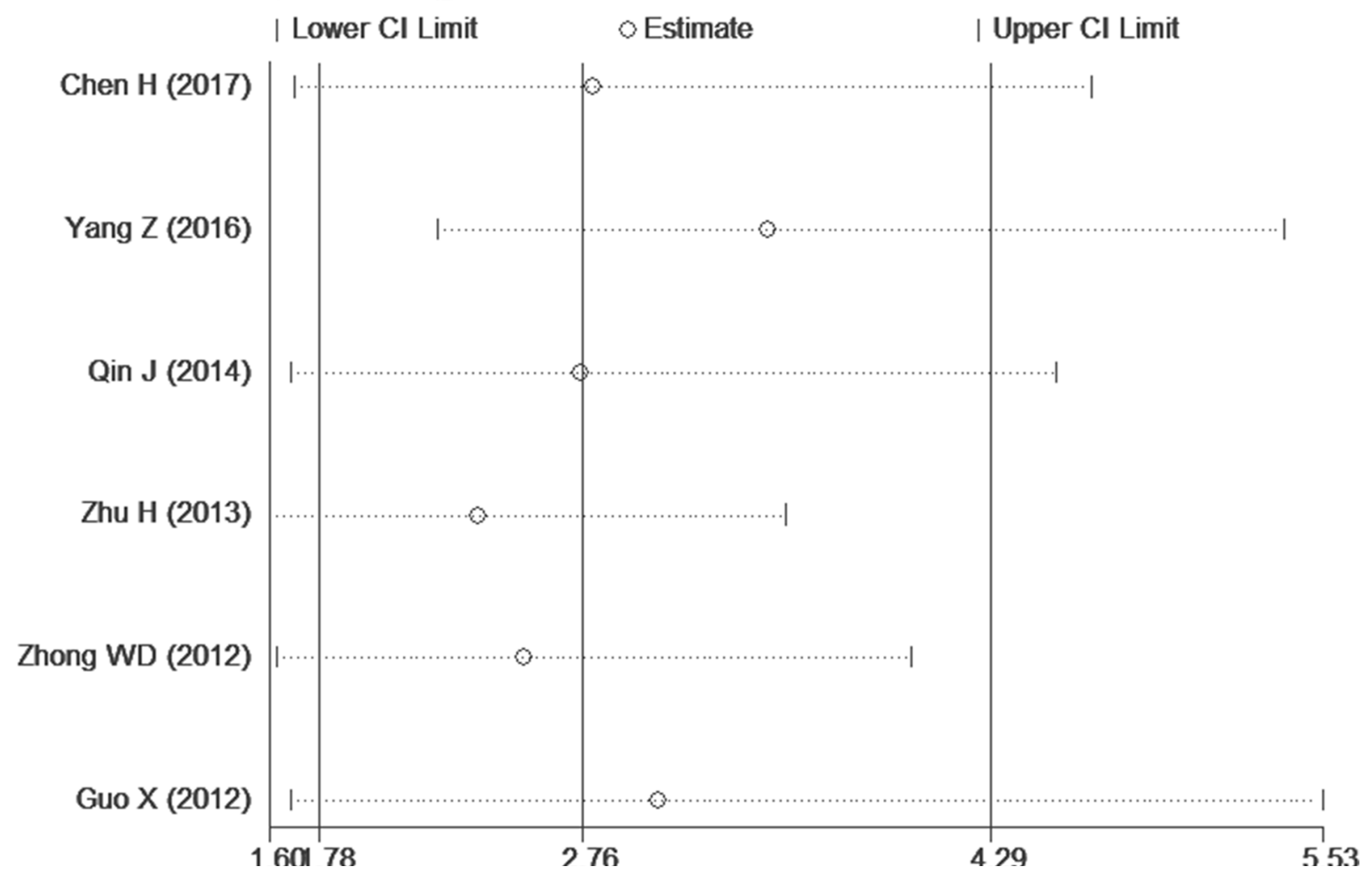

Figure 4: Sensitivity analysis of the OS and DFS in the meta-analysis. 
potential publication bias. In general, concerning these limitations mentioned above, a larger cohort sample size, adjusted individual data and a unified detection method are required to achieve a more persuasive conclusion.

In conclusion, our meta-analysis demonstrated that over-expressed SOX9, as evaluated by IHC, is positively related to poor OS and DFS in human solid tumor patients. Over-expressed SOX9 could be served as a potential biomarker for unfavorable clinicopathological prognostic factors in patients with various solid tumors, suggesting that directly targeting SOX9 could be promising therapeutic approaches for solid malignancies.

\section{MATERIALS AND METHODS}

\section{Literature search strategy}

This systematic review and meta-analysis is reported in accordance with the Preferred Reporting Items for Systematic Review and Meta-Analysis (PRISMA) statement [53]. We performed a thorough search of PubMed, Embase and Web of Science databases for studies measuring expression of SOX9 and survival in patients with solid tumors from 1997 to August 2017.

The search terms included the following key words in various combinations: SOX9, prognosis, prognostic, survival, and overall survival. The hits were restricted to human studies of solid tumors and those published in English. The references list of review and bibliographies were further sifted to identify additional potentially relevant studies to avoid omission due to the electronic search approach.

\section{Study inclusion and exclusion criteria}

The collected studies included in this meta-analysis had to meet the following criteria: (1) a pathological diagnosis of cancer was made; (2) SOX9 expression in patients with any type of tumor was measured via immunohistochemistry; (3) associations of SOX9 expression with OS, DFS or clinicopathological features were described; (4) HRs and 95\% confidence intervals (CIs) were reported or could be calculated (based on the information in the paper); and (5) when the same author reported repeated results from the same population, the most complete report was included. The exclusion criteria for this meta-analysis were as follows: (1) unpublished papers; (2) laboratory articles, reviews and letters; (3) non-English language articles; (4) overlapping articles or ones with duplicate data; (5) articles with only animal experiments; (6) studies without information about survival curves; and (7) SOX9 expression in patients with any type of tumor was analyzed only using RT-PCR method.

\section{Data extraction and quality assessment}

All data were extracted independently by two investigators (Haihua Ruan and Xichuan Li). For each eligible study, the following characteristics were extracted: first author's name, publication year, region, type of cancer, number of patients, patients' ages, followup times, detection methods, cut-off values, survival data (including OS and DFS) and clinicopathological parameters, such as gender, tumor differentiation, tumor size, lymph node metastasis, distant metastasis and clinical stage. For studies that presented only Kaplan-Meier curves was used to extract the survival data. The cut-off values of SOX9 expression were differently indicated among the included studies. Briefly, the percentage scoring (PS) of immunoreactive tumor cells was calculated as follows: 0 $(0 \%), 1(1-25 \%), 2(26-50 \%), 3(51-75 \%)$ and $4(76-$ $100 \%)$. The staining intensity was visually scored and stratified as follows: 0 (negative); 1 (weak); 2 (moderate); and 3 (strong). The immunoreactivity score (IRS) was obtained in some studies by multiplying the percentage and the intensity score.
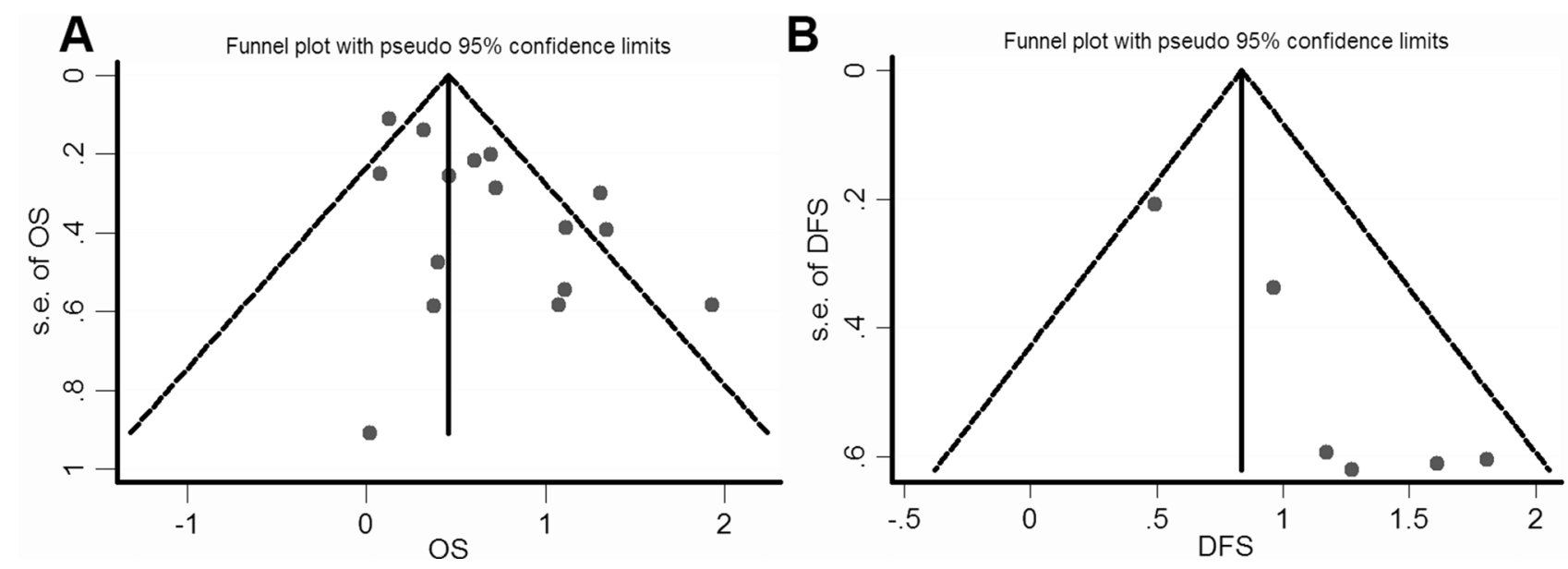

Figure 5: Funnel plot for the assessment of potential publication bias regarding OS and DFS in the meta-analysis. 


\section{Statistical analysis}

This meta-analysis was performed using Stata 12.0 (Stata Corporation, College Station, TX, USA) software. Pooled estimates of HRs and their 95\% CIs were used to estimate the association between SOX9 expression and patients' survival. The chisquared test (Cochrane' s Q test) and I-squared statistical test were used to analyze the heterogeneity between studies. When the result of a Q-test $\left(\mathrm{I}^{2}>50 \%\right.$ or $\left.P<0.05\right)$ indicated heterogeneity, the randomeffects model was used for the meta-analysis. Otherwise, a fixed-effects model was used. HR with its $95 \%$ CI over 1.0 indicated poor prognosis patients with increased SOX 9 expression. Funnel plots were used to graphically represent the publication bias. Begg's (rank correlation) test was adopted to confirm the publication bias. Begg's (rank correlation) and Egger's (regression asymmetry) tests were adopted to confirm the publication bias.

\section{Author contributions}

Haihua Ruan and Xichuan Li conceived and carried out the analysis. Haihua Ruan and Xichuan Li performed literature search, study selection and data extraction. Hongyu Zhang and Shuangyan Hu conducted statistical analysis and quality assessment. Xiaoting Li and Xiaobo Li prepared tables and figures. Haihua Ruan wrote the manuscript. Gang Du revised the manuscript. All authors reviewed the manuscript.

\section{CONFLICTS OF INTEREST}

The authors declare no potential conflicts of interest.

\section{FUNDING}

This work was supported by the National Natural Science Foundation of China (grants 81472681 to X.L. and 31540066 to H.R.).

\section{REFERENCES}

1. Wagner T, Wirth J, Meyer J, Zabel B, Held M, Zimmer J, Pasantes J, Bricarelli FD, Keutel J, Hustert E, Wolf U, Tommerup N, Schempp W, et al. Autosomal sex reversal and campomelic dysplasia are caused by mutations in and around the SRY-related gene SOX9. Cell. 1994; 79:1111-20.

2. Jo A, Denduluri S, Zhang B, Wang Z, Yin L, Yan Z, Kang R, Shi LL, Mok J, Lee MJ, Haydon RC. The versatile functions of Sox9 in development, stem cells, and human diseases. Genes Dis. 2014; 1:149-61. https://doi.org/10.1016/j. gendis.2014.09.004.

3. Kato N, Fukase M, Motoyama T. Expression of a transcription factor, SOX9, in Sertoli-stromal cell tumors of the ovary. Int J Gynecol Pathol. 2004; 23:180-1.
4. Sakamoto H, Mutoh H, Miura Y, Sashikawa M, Yamamoto H, Sugano K. SOX9 Is Highly Expressed in Nonampullary Duodenal Adenoma and Adenocarcinoma in Humans. Gut Liver. 2013; 7:513-8. https://doi.org/10.5009/ gn1.2013.7.5.513.

5. Vidal VP, Ortonne N, Schedl A. SOX9 expression is a general marker of basal cell carcinoma and adnexal-related neoplasms. J Cutan Pathol. 2008; 35:373-9. https://doi. org/10.1111/j.1600-0560.2007.00815.x.

6. Wang H, McKnight NC, Zhang T, Lu ML, Balk SP, Yuan $\mathrm{X}$. SOX9 is expressed in normal prostate basal cells and regulates androgen receptor expression in prostate cancer cells. Cancer Res. 2007; 67:528-36. https://doi. org/10.1158/0008-5472.CAN-06-1672.

7. Wang H, Leav I, Ibaragi S, Wegner M, Hu GF, Lu ML, Balk SP, Yuan X. SOX9 is expressed in human fetal prostate epithelium and enhances prostate cancer invasion. Cancer Res. 2008; 68:1625-30. https://doi.org/10.1158/0008-5472. CAN-07-5915.

8. Ma F, Ye H, He HH, Gerrin SJ, Chen S, Tanenbaum BA, Cai C, Sowalsky AG, He L, Wang H, Balk SP, Yuan X. SOX9 drives WNT pathway activation in prostate cancer. J Clin Invest. 2016; 126:1745-58. https://doi.org/10.1172/ JCI78815.

9. Hong Y, Chen W, Du X, Ning H, Chen H, Shi R, Lin S, Xu $\mathrm{R}$, Zhu J, Wu S, Zhou H. Upregulation of sex-determining region Y-box 9 (SOX9) promotes cell proliferation and tumorigenicity in esophageal squamous cell carcinoma. Oncotarget. 2015; 6:31241-54. https://doi.org/10.18632/ oncotarget.5160.

10. Choi YJ, Song JH, Yoon JH, Choi WS, Nam SW, Lee JY, Park WS. Aberrant expression of SOX9 is associated with gastrokine 1 inactivation in gastric cancers. Gastric Cancer. 2014; 17:247-54. https://doi.org/10.1007/s10120-013-0277-3.

11. Zhang HT, Yang J, Liang GH, Gao XJ, Sang Y, Gui T, Liang ZJ, Tam MS, Zha ZG. Andrographolide Induces Cell Cycle Arrest and Apoptosis of Chondrosarcoma by Targeting TCF-1/SOX9 Axis. J Cell Biochem. 2017; 118: 4575-4586. https://doi.org/10.1002/jcb.26122.

12. Invasion and metastasis. Molecular Biology of Human Cancers: An Advanced Student's Textbook. (Dordrecht: Springer Netherlands), pp. (2005). 193-217.

13. Chen H, Garbutt C, Spentzos D, Choy E, Hornicek FJ, Duan Z. Expression and Therapeutic Potential of SOX9 in Chordoma. Clin Cancer Res. 2017; 23: 5176-5186. https:// doi.org/10.1158/1078-0432.CCR-17-0177.

14. Qi J, Yang Y, Hao P, Xu J. Transcription Factor SOX9 Promotes Osteosarcoma Cell Growth by Repressing Claudin-8 Expression. Tohoku J Exp Med. 2017; 241:5563. https://doi.org/10.1620/tjem.241.55.

15. Liu H, Chen Y, Zhou F, Jie L, Pu L, Ju J, Li F, Dai Z, Wang $\mathrm{X}$, Zhou S. Sox9 regulates hyperexpression of Wnt1 and Fzd1 in human osteosarcoma tissues and cells. Int J Clin Exp Pathol. 2014; 7:4795-805. 
16. Zhu H, Tang J, Tang M, Cai H. Upregulation of SOX9 in osteosarcoma and its association with tumor progression and patients' prognosis. Diagn Pathol. 2013; 8:183. https:// doi.org/10.1186/1746-1596-8-183.

17. Bruun J, Kolberg M, Nesland JM, Svindland A, Nesbakken A, Lothe RA. Prognostic Significance of beta-Catenin, E-Cadherin, and SOX9 in Colorectal Cancer: Results from a Large Population-Representative Series. Front Oncol. 2014; 4:118. https://doi.org/10.3389/fonc.2014.00118.

18. Lu B, Fang Y, Xu J, Wang L, Xu F, Xu E, Huang Q, Lai M. Analysis of SOX9 expression in colorectal cancer. Am J Clin Pathol. 2008; 130:897-904. https://doi.org/10.1309/ AJCPW1W8GJBQGCNI.

19. Yang Z, Cui Y, Ni W, Kim S, Xuan Y. Gli1, a potential regulator of esophageal cancer stem cell, is identified as an independent adverse prognostic factor in esophageal squamous cell carcinoma. J Cancer Res Clin Oncol. 2017; 143:243-54. https://doi.org/10.1007/s00432-016-2273-6.

20. Chakravarty G, Moroz K, Makridakis NM, Lloyd SA, Galvez SE, Canavello PR, Lacey MR, Agrawal K, Mondal D. Prognostic significance of cytoplasmic SOX9 in invasive ductal carcinoma and metastatic breast cancer. Exp Biol Med (Maywood). 2011; 236:145-55. https://doi. org/10.1258/ebm.2010.010086.

21. Lapierre M, Linares A, Dalvai M, Duraffourd C, Bonnet S, Boulahtouf A, Rodriguez C, Jalaguier S, Assou S, Orsetti B, Balaguer P, Maudelonde T, Blache P, et al. Histone deacetylase 9 regulates breast cancer cell proliferation and the response to histone deacetylase inhibitors. Oncotarget. 2016; 7:19693-708. https://doi.org/10.18632/oncotarget.7564.

22. Riemenschnitter C, Teleki I, Tischler V, Guo W, Varga Z. Stability and prognostic value of Slug, Sox9 and Sox 10 expression in breast cancers treated with neoadjuvant chemotherapy. Springerplus. 2013; 2:695. https://doi. org/10.1186/2193-1801-2-695.

23. Willis S, De P, Dey N, Long B, Young B, Sparano JA, Wang V, Davidson NE, Leyland-Jones BR. Enriched transcription factor signatures in triple negative breast cancer indicates possible targeted therapies with existing drugs. Meta Gene. 2015; 4:129-41. https://doi.org/10.1016/j. mgene.2015.04.002.

24. Liu C, Liu L, Chen X, Cheng J, Zhang H, Shen J, Shan J, Xu Y, Yang Z, Lai M, Qian C. Sox9 regulates self-renewal and tumorigenicity by promoting symmetrical cell division of cancer stem cells in hepatocellular carcinoma. Hepatology. 2016; 64:117-29. https://doi.org/10.1002/hep.28509.

25. Xue TC, Zhang L, Ren ZG, Chen RX, Cui JF, Ge NL, Ye SL. Sex-determination gene SRY potentially associates with poor prognosis but not sex bias in hepatocellular carcinoma. Dig Dis Sci. 2015; 60:427-35. https://doi.org/10.1007/ s10620-014-3377-y.

26. Gao J, Zhang JY, Li YH, Ren F. Decreased expression of SOX9 indicates a better prognosis and inhibits the growth of glioma cells by inducing cell cycle arrest. Int J Clin Exp Pathol. 2015; 8:10130-8.
27. Li J, Wang L, Liu Z, Zu C, Xing F, Yang P, Yang Y, Dang X, Wang K. MicroRNA-494 inhibits cell proliferation and invasion of chondrosarcoma cells in vivo and in vitro by directly targeting SOX9. Oncotarget. 2015; 6:26216-29. https://doi.org/10.18632/oncotarget.4460.

28. Elimova E, Wadhwa R, Shiozaki H, Sudo K, Estrella JS, Badgwell BD, Das P, Matamoros A Jr, Song S, Ajani JA. Molecular biomarkers in gastric cancer. J Natl Compr Canc Netw. 2015; 13:e19-29.

29. Shao CM, Shao QS, Yao HB, Zhao ZK, Xu J, Zhao ZS, Tao HQ. [Association of SOX9 expression and prognosis in patients with gastric cancer]. [Article in Chinese]. Zhonghua Wei Chang Wai Ke Za Zhi. 2012; 15:736-9.

30. Wang Q, Zhang J, Zhong YF, Cong Y, Lin D. [SOX9 expression correlates with microvascular density, progress and prognosis in gastric cancer patients]. [Article in Chinese]. Zhonghua Bing Li Xue Za Zhi. 2012; 41:848-9. https://doi.org/10.3760/cma.j.issn.0529-5807.2012.12.014.

31. Cheng PF, Shakhova O, Widmer DS, Eichhoff OM, Zingg D, Frommel SC, Belloni B, Raaijmakers MI, Goldinger SM, Santoro R, Hemmi S, Sommer L, Dummer R, et al. Methylation-dependent SOX9 expression mediates invasion in human melanoma cells and is a negative prognostic factor in advanced melanoma. Genome Biol. 2015; 16: 42. https://doi.org/10.1186/s13059-015-0594-4.

32. Xia S, Feng Z, Qi X, Yin Y, Jin J, Wu Y, Wu H, Feng Y, Tao M. Clinical implication of Sox 9 and activated Akt expression in pancreatic ductal adenocarcinoma. Med Oncol. 2015; 32:358. https://doi.org/10.1007/s12032-0140358-0.

33. Raspaglio G, Petrillo M, Martinelli E, Li Puma DD, Mariani M, De Donato M, Filippetti F, Mozzetti S, Prislei S, Zannoni GF, Scambia G, Ferlini C. Sox9 and Hif-2alpha regulate TUBB3 gene expression and affect ovarian cancer aggressiveness. Gene. 2014; 542:173-81. https://doi. org/10.1016/j.gene.2014.03.037.

34. Qin GQ, He HC, Han ZD, Liang YX, Yang SB, Huang YQ, Zhou L, Fu H, Li JX, Jiang FN, Zhong WD. Combined overexpression of HIVEP3 and SOX9 predicts unfavorable biochemical recurrence-free survival in patients with prostate cancer. Onco Targets Ther. 2014; 7:137-46. https:// doi.org/10.2147/OTT.S55432.

35. Zhong WD, Qin GQ, Dai QS, Han ZD, Chen SM, Ling XH, Fu X, Cai C, Chen JH, Chen XB, Lin ZY, Deng YH, Wu SL, et al. SOXs in human prostate cancer: implication as progression and prognosis factors. BMC Cancer. 2012; 12:248. https://doi.org/10.1186/1471-2407-12-248.

36. Zhou CH, Ye LP, Ye SX, Li Y, Zhang XY, Xu XY, Gong LY. Clinical significance of SOX9 in human non-small cell lung cancer progression and overall patient survival. J Exp Clin Cancer Res. 2012; 31:18. https://doi.org/10.1186/17569966-31-18.

37. Pomp V, Leo C, Mauracher A, Korol D, Guo W, Varga Z. Differential expression of epithelial-mesenchymal transition and stem cell markers in intrinsic subtypes of breast cancer. 
Breast Cancer Res Treat. 2015; 154:45-55. https://doi. org/10.1007/s10549-015-3598-6.

38. Guo X, Xiong L, Sun T, Peng R, Zou L, Zhu H, Zhang J, Li H, Zhao J. Expression features of SOX9 associate with tumor progression and poor prognosis of hepatocellular carcinoma. Diagn Pathol. 2012; 7:44. https://doi. org/10.1186/1746-1596-7-44.

39. Matsushima H, Kuroki T, Kitasato A, Adachi T, Tanaka T, Hirabaru M, Hirayama T, Kuroshima N, Hidaka M, Soyama A, Takatsuki M, Kinoshita N, Sano K, et al. Sox9 expression in carcinogenesis and its clinical significance in intrahepatic cholangiocarcinoma. Dig Liver Dis. 2015; 47:1067-75. https://doi.org/10.1016/j.dld.2015.08.003.

40. Yun JY, Kim YA, Choe JY, Min H, Lee KS, Jung Y, Oh $\mathrm{S}$, Kim JE. Expression of cancer stem cell markers is more frequent in anaplastic thyroid carcinoma compared to papillary thyroid carcinoma and is related to adverse clinical outcome. J Clin Pathol. 2014; 67:125-33. https:// doi.org/10.1136/jclinpath-2013-201711.

41. Candy PA, Phillips MR, Redfern AD, Colley SM, Davidson JA, Stuart LM, Wood BA, Zeps N, Leedman PJ. Notch-induced transcription factors are predictive of survival and 5-fluorouracil response in colorectal cancer patients. Br J Cancer. 2013; 109:1023-30. https://doi.org/10.1038/bjc.2013.431.

42. Sun M, Uozaki H, Hino R, Kunita A, Shinozaki A, Ushiku T, Hibiya T, Takeshita K, Isogai M, Takada K, Fukayama M. SOX9 expression and its methylation status in gastric cancer. Virchows Arch. 2012; 460:271-9. https://doi. org/10.1007/s00428-012-1201-7.

43. Sinclair AH, Berta P, Palmer MS, Hawkins JR, Griffiths BL, Smith MJ, Foster JW, Frischauf AM, Lovell-Badge R, Goodfellow PN. A gene from the human sex-determining region encodes a protein with homology to a conserved DNA-binding motif. Nature. 1990; 346:240-4. https://doi. org/10.1038/346240a0.

44. Chaboissier MC, Kobayashi A, Vidal VI, Lutzkendorf S, van de Kant HJ, Wegner M, de Rooij DG, Behringer RR, Schedl A. Functional analysis of Sox 8 and Sox9 during sex determination in the mouse. Development. 2004; 131:1891901. https://doi.org/10.1242/dev.01087.

45. Koizumi K, Tanimoto T, Kubota Y, Kitahata S. Enzymatic synthesis, isolation, and analysis of novel alpha- and betagalactosyl-cycloisomalto-octaoses. Carbohydr Res. 1997; 305:393-400.

46. Yoon JH, Kang YH, Choi YJ, Park IS, Nam SW, Lee JY, Lee YS, Park WS. Gastrokine 1 functions as a tumor suppressor by inhibition of epithelial-mesenchymal transition in gastric cancers. J Cancer Res Clin Oncol. 2011; 137:1697-704. https://doi.org/10.1007/s00432-011-1051-8.

47. Kawai T, Yasuchika K, Ishii T, Miyauchi Y, Kojima H, Yamaoka R, Katayama H, Yoshitoshi EY, Ogiso S, Kita S, Yasuda K, Fukumitsu K, Komori J, et al. SOX9 is a novel cancer stem cell marker surrogated by osteopontin in human hepatocellular carcinoma. Sci Rep. 2016; 6:30489. https:// doi.org/10.1038/srep30489.

48. Li XM, Piao YJ, Sohn KC, Ha JM, Im M, Seo YJ, Whang $\mathrm{KU}$, Lee JH, Lee Y, Kim CD. Sox9 is a beta-cateninregulated transcription factor that enhances the colonyforming activity of squamous cell carcinoma cells. Mol Med Rep. 2016; 14:337-42. https://doi.org/10.3892/ mmr.2016.5210.

49. Cui Y, Cui CA, Yang ZT, Ni WD, Jin Y, Xuan YH. Gli1 expression in cancer stem-like cells predicts poor prognosis in patients with lung squamous cell carcinoma. Exp Mol Pathol. 2017; 102:347-53. https://doi.org/10.1016/j. yexmp.2017.03.004.

50. Rahmoun M, Lavery R, Laurent-Chaballier S, Bellora N, Philip GK, Rossitto M, Symon A, Pailhoux E, Cammas F, Chung J, Bagheri-Fam S, Murphy M, Bardwell V, et al. In mammalian foetal testes, SOX9 regulates expression of its target genes by binding to genomic regions with conserved signatures. Nucleic Acids Res. 2017; 45:7191-211. https:// doi.org/10.1093/nar/gkx328.

51. Qian Y, Xia S, Feng Z. Sox9 mediated transcriptional activation of FOXK2 is critical for colorectal cancer cells proliferation. Biochem Biophys Res Commun. 2017; 483:475-81. https://doi.org/10.1016/j.bbrc.2016.12.119.

52. Thomsen MK, Ambroisine L, Wynn S, Cheah KS, Foster CS, Fisher G, Berney DM, Moller H, Reuter VE, Scardino P, Cuzick J, Ragavan N, Singh PB, et al. SOX9 elevation in the prostate promotes proliferation and cooperates with PTEN loss to drive tumor formation. Cancer Res. 2010; 70:979-87. https://doi.org/10.1158/0008-5472.CAN-092370.

53. Liberati A, Altman DG, Tetzlaff J, Mulrow C, Gotzsche PC, Ioannidis JP, Clarke M, Devereaux PJ, Kleijnen J, Moher D. The PRISMA statement for reporting systematic reviews and meta-analyses of studies that evaluate health care interventions: explanation and elaboration. Ann Intern Med. 2009; 151:W65-94. 